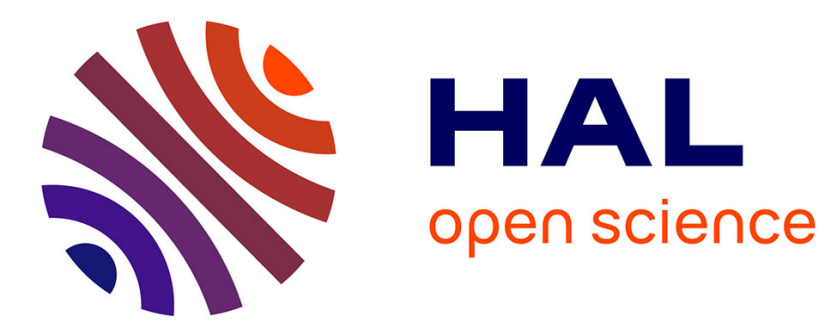

\title{
Electric current based power line communication for plug-load device auto identification
}

Hiroyuki Ikegami, Manabu Tsukada, Hideya Ochiai, Hideaki Nii, Hiroshi Esaki

\section{To cite this version:}

Hiroyuki Ikegami, Manabu Tsukada, Hideya Ochiai, Hideaki Nii, Hiroshi Esaki. Electric current based power line communication for plug-load device auto identification. 6th IEEE International Conference on Smart Grid Communications (SmartGridComm 2015), Nov 2015, Miami, FL, United States. 10.1109/SmartGridComm.2015.7436287 . hal-01317105

\section{HAL Id: hal-01317105 \\ https://hal.science/hal-01317105}

Submitted on 18 May 2016

HAL is a multi-disciplinary open access archive for the deposit and dissemination of scientific research documents, whether they are published or not. The documents may come from teaching and research institutions in France or abroad, or from public or private research centers.
L'archive ouverte pluridisciplinaire HAL, est destinée au dépôt et à la diffusion de documents scientifiques de niveau recherche, publiés ou non, émanant des établissements d'enseignement et de recherche français ou étrangers, des laboratoires publics ou privés. 


\title{
Electric Current Based Power Line Communication for Plug-Load Device Auto Identification
}

\author{
Hiroyuki Ikegami*, Manabu Tsukada*, Hideya Ochiai*, Hideaki Nii ${ }^{\dagger}$ and Hiroshi Esaki* \\ *the University of Tokyo, Japan \\ Email: \{ikegam, tsukada, jo2lxq\}@hongo.wide.ad.jp, hiroshi@wide.ad.jp \\ †IIJ Innovation Institute, Japan \\ Email: h-nii@iij.ad.jp
}

\begin{abstract}
A smart building has an outlet-level power meters for managing the energy consumption of plug-load devices - the devices or appliances connected to the outlets. Identification of the connected devices used to be made by system operators in the traditional building energy management systems (BEMS), but it is no more realistic for such fine-grained power monitoring applications. This paper proposes a new communication method electric current based power line communication (ECPLC), which enables automatic identification of plug-load devices without the intervention of system operators. Using ECPLC, a device transmits bit sequences that show its identifier on the electric current flow, which propagates only to the outlet from the connected device. We have designed a transmitter and a receiver of ECPLC with studying the electric model that enables the communication. We have also implemented them, and carried out experiments on varieties of cases. The results show that ECPLC performs very well in typical application scenarios, indicating that it is useful for automatic identification of plug-load devices.
\end{abstract}

Keywords-Power Line Communication, Smart Buildings, Building Energy Management System.

\section{INTRODUCTION}

A smart building has outlet-level power meters to allow fine-grained monitoring of energy consumption of devices or appliances - which are called "plug-load devices" in this paper. Smart building needs identification of the plug-load devices connected to the outlets to manage the consumption. Building operators used to put the connection information by hand in a traditional building energy management system (BEMS), however this approach reaches the limit. Some devices are portable, which may connect to any outlets and BEMS cannot follow the change without intervention of the building operators.

Automatic identification of plug-load devices is necessary to outlet-level energy management. Inspired by the works of non-intrusive load monitoring (NILM[10][12]), researchers have studied on the "features" of energy consumption [3][9] (i.e., pattern of energy usage), trying to identify the device connected to the outlet. We call this approach as passive scheme. Passive approach can estimate the types of the devices by the observation of the energy consumption. However, the approach cannot easily "identify" the device itself if they have many similar devices. Active identification schemes are probably essential so as to precisely perform auto identification in such a scenario.

In this paper, we propose a new power line communication, named electric current based power line communication (ECPLC). ECPLC can transmit bit sequences using "electric current", which propagates on a closed circuit - making a loop. In the proposal, each device periodically transmits its identifier on its power line, and the outlet can receive the identifier of the connected device. In this way, we can achieve automatic identification of plug-load devices.

ECPLC is totally different from the well-known PLCs[1][8][7]. They transmit signals on the voltage of the power line, which we say electric voltage based PLC (EVPLC). EVPLC cannot be used due to the nature of the voltage propagation. The signals of EVPLC transmitted from a device would reach all the outlets, therefore we would not be able to identify where it is connected. The signals of ECPLC from a device will reach the sole outlet, and we can recognize that the device is connected to the outlet. The proposed ECPLC enables immediate and precise identification.

This paper focuses on the basic principles of ECPLC. We assume one-to-one (device-to-outlet) simplex communication, propose the design of the transmitter and the receiver of ECPLC and develop a signal propagation model. We have implemented the ECPLC transmitter and the receiver and evaluated the performance considering typical building deployment scenario. The following other practical works are out of focus in this paper: collision avoidance, duplex communication, design of identifiers and system implementation.

The rest of the paper is organized as follows. Section II highlights the works related to the domain of plug-load auto identification and power line communication. Section III explains the proposal, ECPLC. the ECPLC transmitter and the ECPLC receiver are also introduced. Section IV provides the evaluation of ECPLC. In Section V, we discuss the result of the experiment and lists the future work. Finally, Section VI concludes the paper.

\section{RELATED WORK}

Plug-load device identification is a necessary task for power consumption measurement as described in the introduction. Administrator of power measurement equipment manually writes up the association such as which plug-load device is monitored by which point of power metering typically. For instance, power distribution unit (PDU) in a data center and smart power strip (SPS) in a building employ the manual manner. Contrary to manual, some automatic technologies are also proposed. Electric vehicle (EV) and Smart Plug have the auto identification functionality by design such as [2] and [11]. These dedicated systems have another communication channel 
for the identification by modification of $\mathrm{AC}$ plug and power outlet.

Some researchers focused power metering itself to identify plug-load device based on time-series voltage, current and phase data. They are trying plug-load device auto identification with data analysis technology. This is a hot research topic, called as non intrusive load monitoring (NILM), includes plug-load auto identification function typically [10][12]. Other works such as [3], [4] and [9] also focus on plug-load auto identification from time-series data analysis.

Power line communication is conventionally voltage based, which modulates data to high frequency electric voltage and uses a power line system of a building as a bus network (electric voltage based power line communication, EVPLC). EVPLC is widely operated such as X10, HD-PLC[1] and G3PLC [8]. There are an enormous number of studies[7]. EVPLC easily expands the in-building computer network because of the nature of electric voltage. Wall outlets are connected as parallel, therefore voltage signal of EVPLC propagates all outlets. However EVPLC does not aim the issue of plug-load device identification.

TWACS[6][5] is one of PLC technology and TWACS uses current flow for data communication on a power line. TWACS focuses on transmission distance of current based communication because TWACS is designed for auto power metering from the power company to their customer. Inbound communication of TWACS, from the power meter to a master device, uses current flow to transmit data in order to enable long-distance transmission around tens kilometers.

\section{Electric Current BAsed Power Line COMMUNICATION}

This section explains the proposal, electric current-based power line communication (ECPLC). ECPLC is a new type of communication method designed for plug-load device auto identification system. The proposal includes circuit designs of ECPLC transmitter and receiver. An electrical model of the ECPLC communication system is also introduced.

\section{A. Plug-load device auto identification system with ECPLC}

The overview of the proposed system is shown at Fig. 1. All of plug-load devices transmit an own device identifier such as an IPv6 address and Ethernet MAC address to smart power strips, a smart outlet or smart meters by electric current flow. These smart devices receive the identifier and then identify own target devices. The result of this identification may send to a center storage server, which saves power consumption data and the server keeps association correctly.

This paper uses terminologies, which are smart meter, smart outlet and smart power strip, for the following contents. The Smart meter indicates a power consumption metering unit for the root of power lines, which is located on the electrical panel typically. Smart outlet indicates a wall outlet with power consumption metering and smart power strip is an extension power cable with the metering function.

ECPLC is suitable for the system, because signals of ECPLC propagate to upstream of a power line by consuming electricity in ECPLC transmitter and other power outlets which

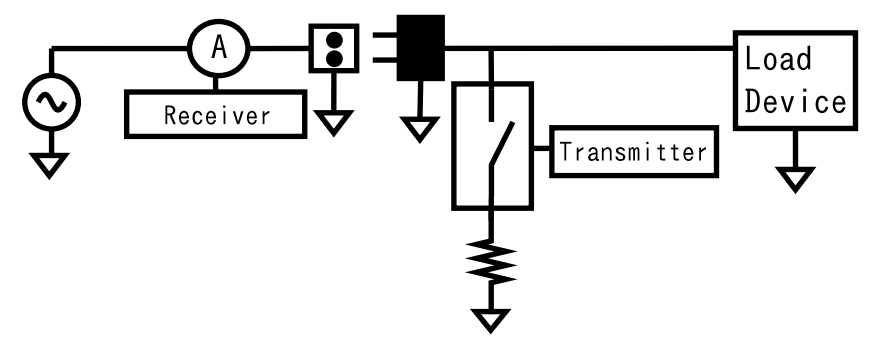

Fig. 1. Plug-load device auto identification system based on electric current flow based communication

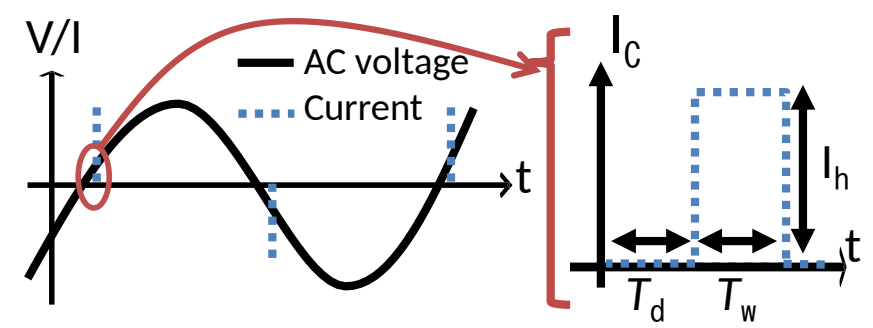

Fig. 2. ECPLC waveform: Current pulse at voltage zero crossing point

are connected as paralell do not receive signals based on physics of current flow unlike EVPLC.

\section{B. ECPLC waveform}

Fig. 2 shows a fundamental idea to represent binary value ( 1 or 0 ) on current flow and the parameters, $T_{d}, T_{w}$ and $I_{h}$. ECPLC makes a short term current pulse at voltage zero crossing point to send " 1 " and no pulse at the point means " 0 ". In another word, it is binary coded pulse width modulation on voltage zero crossing point. Using a zero crossing point makes good signal-to-noise ratio, because almost none of the plugload devices consume electricity at the time range.

This proposed waveform has three parameters, which are $T_{d}, T_{w}$ and $I_{h}$ in Fig. 2. $T_{d}$ stands for a delay before starting a pulse. $T_{w}$ and $I_{h}$ are the pulse width and height.

\section{ECPLC Transmitter}

The proposed transmission method is based on the constant current source circuit. Fig. 3 shows an important part of the proposed transmitter circuit. An AC plug is a power source and an $\mathrm{AC}$ input capable photocoupler is placed on the $\mathrm{AC}$ line in order to catch zero crossing point for a micro computing unit (MCU). A diode bridge is placed on same AC line and $\mathrm{AC}$ voltage is converted to full rectified wave. The output of the diode bridge connects to GND and the gate of $\operatorname{Tr}_{1}$, an NPN transistor. An operational amplifier (op-amp), $\operatorname{Tr}_{1}$ and a resistor compose the constant current source circuit. The MCU controls the constant current source circuit based on the output of the photocoupler.

$V_{i n}, V_{+}, V_{-}$and $V_{R}$ stand for the input voltage of the constant current source, the positive input voltage of the opamp, the negative input voltage of the op-amp and the output voltage of the op-amp respectively as shown in Fig. 3. The op-amp makes constant current flow by $V_{i n}$. The op-amp controls own output voltage to maintain $V_{+}=V_{-}$. $V_{\text {in }}$ directly connects to $V_{+}$and $V_{-}$also directly means $V_{R}$, thus, $V_{R}=V_{i n}$. Finally, $I_{h}$ is determined as $V_{i n} / R_{C}$ because of $\mathrm{V}_{R}=V_{i n}$. 


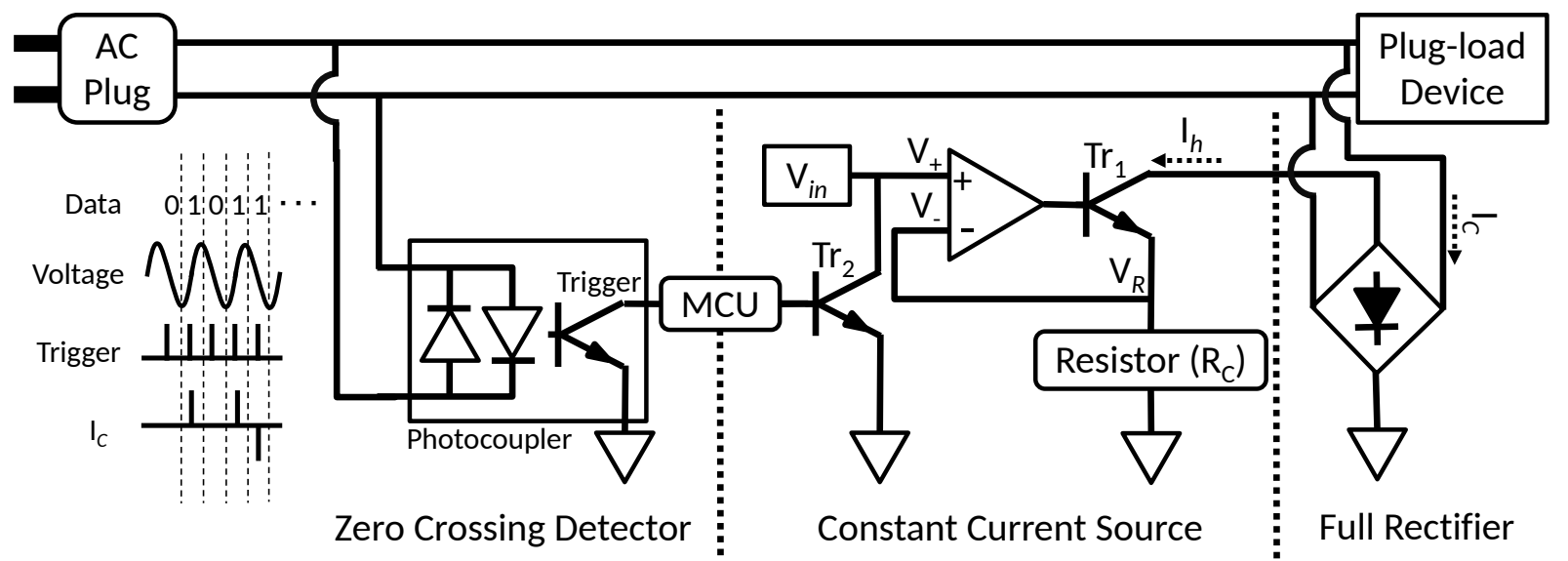

Fig. 3. ECPLC Transmitter: the transmitter makes current pulse at voltage zerocrossing timing to represent data to be transmitted.

The MCU makes $V_{+}=0$ except zero crossing timing by turning on $\mathrm{Tr}_{2}$. Light emitting diodes (LED) of the photocoupler are connected to the AC line. On the low voltage timing, LEDs do not emit light and the photo transistor goes to turn off. When the MCU caches the zero crossing from the photocoupler, the MCU waits $T_{d}$ and turns off $\operatorname{Tr}_{2}$ to make constant current flow as $I_{h}$ in $T_{w}$ period.

\section{ECPLC Receiver}

We propose two types of ECPLC receivers, which are with voltage (normal receiver) and without voltage (simple receiver), because of the real situation on deployment of smart outlets, smart plug strips and smart meters. Smart outlets and smart plug strips have a voltage sensing unit to calculate power consumption accurately. However, at the smart meter level, some conventional smart meters do not have voltage sensing. The smart meters assumes that power factor $=1$ and simply provides apparent power (VA).

The receivers' circuit is shown at Fig. 4. The receivers measures current flow by a current transformer (CT) and catchs the edges of current flow in order to detect current pulses. The conditions to accept a pulse are that height of the edge is $I_{h}$ with under $30 \%$ errors and the time difference between two edges is $T_{w}$ with under $10 \%$ errors. Detecting a pulse means the receiver gets 1 .

The simple receiver measures current flow only as shown as Fig. 4. Therefore, to receive 0 , the receiver interpolates 0 based on the time difference between " 1 " to " 1 ". For instance, if the receiver receives two current pulses at $t$ and $t+32$ milliseconds. Two "0" are interpolated on between 1 on $t$ and 1 on $t+32$ milliseconds, because there are two times zero crossing on $50 \mathrm{~Hz}$ power systems. The normal receiver does not have this function because the receiver detects the time slot of ECPLC transmission by the voltage zero crossing detector.

\section{E. Model of ECPLC signal transmission}

Here, we introduce an electrical model of the ECPLC communication system shown at Fig. 5. $R_{P 1}$ stands for resistances on in-building power lines and $R_{P 2}$ stands for the extension power cable from the ECPLC receiver to the ECPLC transmitter. $R_{I N T}$ is an internal resistance of the transmitter.

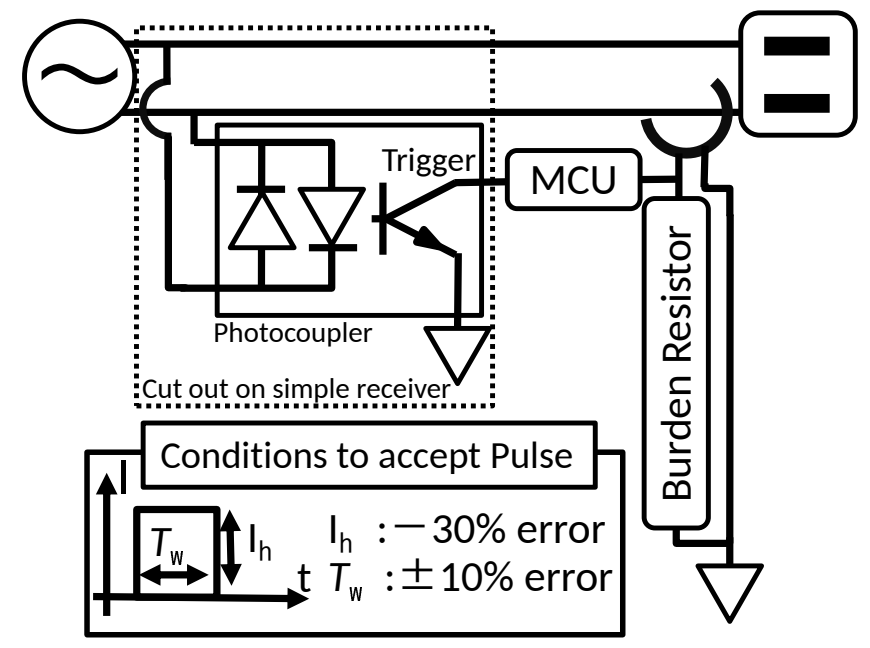

Fig. 4. ECPLC Receiver: Receiver measures current flow by the current transformer and detects transmitted pulse based on the conditions. Simple receiver does not have the zero crossing detector.

The transmitter consists of the switch, the constant current source and the internal resistance. The current source makes $I_{h}$ in $T_{d}<t<T_{d}+T_{w}$ period. $V_{A C}(t)$ is determined as Eq. 1. The ECPLC signal transmission can be considered on this resistance only model circuit without capacitance and inductance of cables, because of frequency of ECPLC. The width of the current pulse is assumed from 100 to 500 microseconds and this corresponds $5 \mathrm{kHz}$ to $1 \mathrm{KHz}$, therefore the model can ignore them.

$$
V_{A C}(t)=\sqrt{2} V_{\text {sys }} \sin \left(2 \pi f_{\text {sys }} t\right)
$$

A time-series ECPLC current flow function, $I_{C}(t)$, can be determined as Eq. 2. $V_{\text {sys }}$ and $f_{\text {sys }}$ stands for the AC power supply voltage and its frequency respectively. $R_{P 1}$ is under $1.5 \Omega$ in $100 \mathrm{~V}$ power system like Japan because voltage must be between 90 to $110 \mathrm{~V}$ with maximum current $15 \mathrm{~A}$. $10 \mathrm{~V}$ voltage drop with 15 A means $1.5 \Omega$. $R_{I N T}$ depends on the transmitter implementation. For instance, in the experimental implementation of this paper, the resistance is $0.5 \Omega$. 


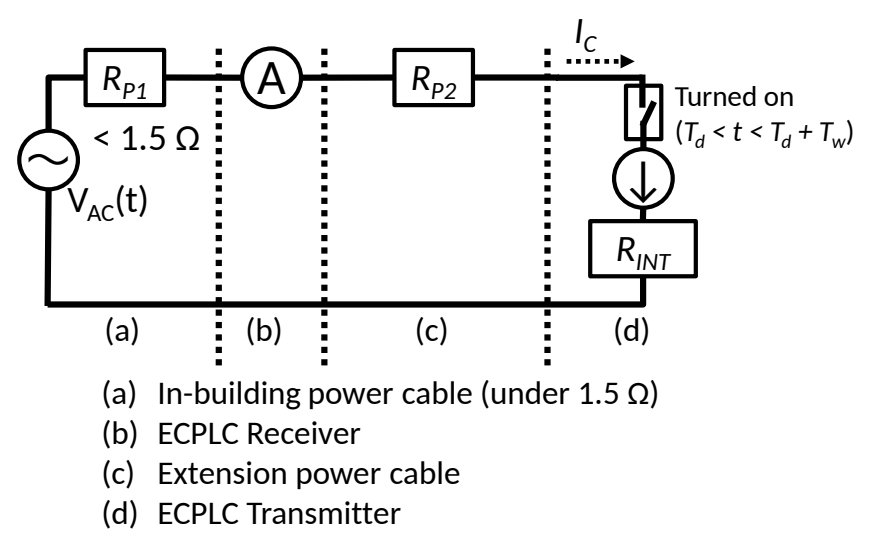

Fig. 5. Electrical model circuit for ECPLC communication system

$$
I_{C}(t)= \begin{cases}0 & \left(t<T_{d}\right) \\ 0 & \left(T_{d}+T_{w}<t\right) \\ \min \left\{\frac{V_{A C}(t)}{R_{I N T}+R_{P 1}+R_{P 2}}, I_{h}\right\} & (\text { otherwise })\end{cases}
$$

The maximum pulse height is capped by $T_{d} . T_{d}$ is determined from $I_{h}$ as Eq. 3, This equation is deformed from Eq. 2 with $I_{C}(t)=I_{h}$. From the equation, for instance, the case of $5.5 \mathrm{~A}$ for $I_{h}$ requires 250 microsecond delay as $T_{d}$ with $R_{P 2}=0, R_{I N T}=0.5 \Omega$ and $R_{P 1}=1.5 \Omega$.

$$
T_{d} \geq \frac{1}{2 \pi f} \arcsin \left(\frac{R_{I N T}+R_{P 1}+R_{P 2}}{\sqrt{2} V_{\text {sys }}} I_{h}\right)
$$

$T_{w}$ is considered from the receiver's analog-to-digital converter (ADC) sampling frequency, $f_{A D C}$, as shown in Eq. 4. For instance, if the frequency is $60 \mathrm{kHz}, T_{w}$ is over 167 microseconds, because of the receiver design. We defined the acceptable error rate is $10 \%$ as defined in the previous section. \pm 16.7 microseconds error should be occurred by $60 \mathrm{kHz}$ sampling, thus $T_{w}$ should be over 167 microseconds.

$$
T_{w} \geq \frac{1}{f_{A D C}} \times 10
$$

$I_{h}$ is decided by the designed power consumption to send " 1 " of the transmitter. The maximum power consumption, $W_{h}$, is calculated from Eq. 5. The reason of the multiplication by $2 f_{\text {sys }}$ is that the time range from $T_{d}$ to $T_{d}+T_{w}$ is for a half part of a wave. From the equation, $I_{h}$ can be determined by power consumption. For instance, on the case of power consumption should be under $3 \mathrm{~W}$, the ADC frequency of the receiver is 60 $\mathrm{kHz}$ and the other parameters are same as above paragraphs, hence up to $6 \mathrm{~A}$ can be used.

$$
W_{h}=\int_{T_{d}}^{T_{d}+T_{w}} I_{C}(t) V_{A C}(t) \cdot d t \times 2 f_{s y s}
$$

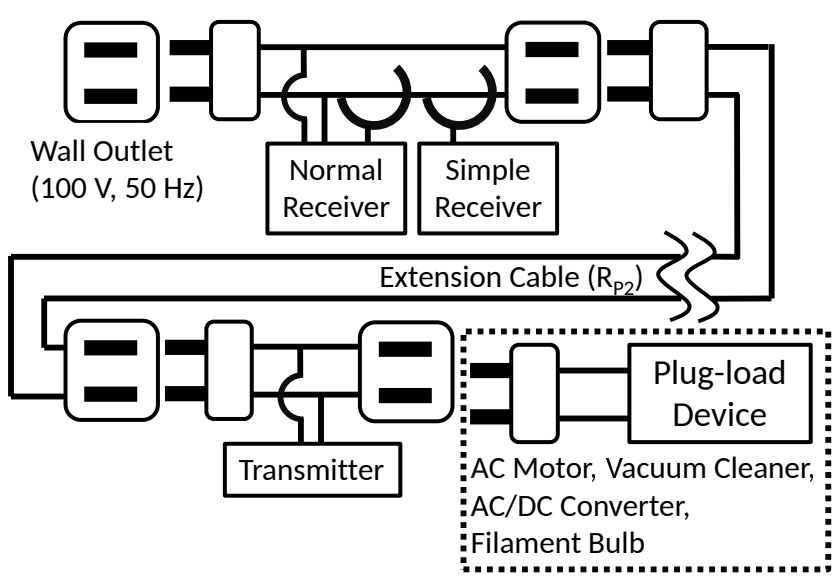

Fig. 6. Experiment system: the transmitter connects two receivers, normal receiver and simple receiver, via the extension power cable.

\section{Evaluation}

This section describes the prototype implementation of ECPLC and experimentation to evaluate characteristics of ECPLC. We conducted two experiments. First one is to investigate the effect of the resistance of the extension cable, $R_{P 2}$. Second one is impact of load current flow of the plug-load device itself to the communication success rate.

\section{A. Experiment Setting}

Experiment system is a one-to-one connection of the transmitter and two receivers, normal receiver and simple receiver, as shown in Fig. 6. The power cable drum between the transmitter and the receivers is the extension power cable for $R_{P 2}$. The power source is the wall outlet of our building (50 $\mathrm{Hz}$ and $100 \mathrm{~V}$ ).

Hardware configurations of the experimentation are summarized in TABLE. I. MCUs (model: ATMEL ATmega328P) is used for the receivers. The MCU takes a current value from the ADC and demodulates binary data. The receivers use a CT (model: U_RD CTL-10-CLS) with $1 \mathrm{k} \Omega$ burden resistor as the current sensor. The normal receiver uses a photocoupler (model: Fairchild Semiconductor FOD814A300W) as the zero crossing detector. The transmitter makes constant current by the op-amp (model: HTC LM358N) and two transistors (model: TOSHIBA 2SC5200, 2SC3421) as a Darlington pair. The output of the op-amp connects to the Darlington circuit to make current pulse. The MCU (same as receivers) connects to the op-amp and works as the binary pulse width modulator.

To evaluate the ECPLC experiment system, here, we determine example parameters as $T_{d}=250$ microseconds, $I_{h}=5.5 \mathrm{~A}$ and $T_{w}=350$ microseconds. To calculate a success rate, the transmitter sends pseudo-random 32bit sequence 200 times, 800 bytes. Success rate is defined as if the receiver demodulates the 32bit chunk correctly that it means success, but if there is any bit errors that it is fail. In addition, throughput of the implementation is $100 \mathrm{bps}$ because of the power system frequency $50 \mathrm{~Hz}$.

\section{B. Pulse width decreasing on extension power cables}

Fig. 7(a) shows shapes of current pulse on the three cables which are $100 \mathrm{~m}(0.98 \Omega), 60 \mathrm{~m}(0.64 \Omega)$ and $4 \mathrm{~m}(0.10 \Omega)$ 
TABLE I. HARDWARE CONFIGURATIONS OF THE EXPERIMENTATION

\begin{tabular}{c|c}
\multicolumn{1}{c||}{ Simple Receiver } & \\
\hline \hline MCU & ATMEL ATmega328P, 16MHz, 2KB SRAM \\
\hline ADC & MCU internal, 60 kHz \\
\hline Current sensing & CT, U_RD CTL-10-CLS \\
\hline Burden resistor & $1 \mathrm{k} \Omega$ \\
\hline \hline Normal Receiver & ATMEL ATmega328P, 16MHz, 2KB SRAM \\
\hline MCU & MCU internal, 60 kHz \\
\hline ADC & $1 \mathrm{k} \Omega$ \\
\hline Current sensing & Fairchild Semiconductor FOD814A300W \\
\hline Burden resistor & ATMEL ATmega328P, 16MHz, 2KB SRAM \\
\hline \hline Voltage sensing & Fairchild Semiconductor FOD814A300W \\
\hline \hline Transmitter & HTC LM358N, TOSHIBA 2SC5200, 2SC3421 \\
\hline MCU & $0.39 \Omega$ \\
\hline Voltage sensing &
\end{tabular}

(a) Pulse shapes on the cables

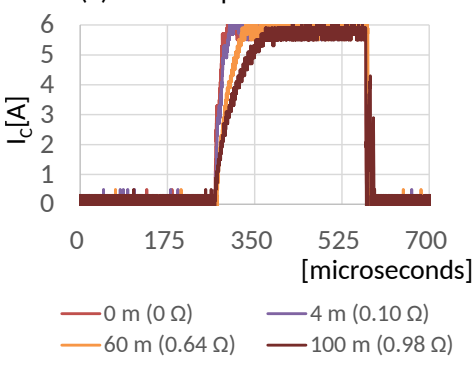

(b) Resistance vs success rate

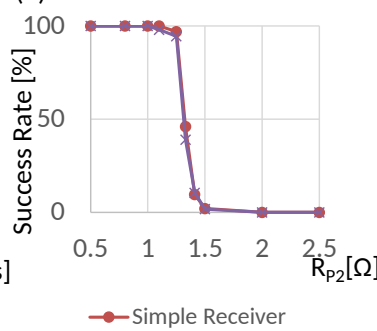

$\rightarrow$ Normal Receiver

Fig. 7. (a) Shapes of current pulse on different cables. (b) Communication success rate versus the resistance of extension cable.

respectively. We changed $\mathrm{R}_{P 2}$ on Fig. 5 by the three cables. The series named as $0 \mathrm{~m}$ means that the transmitter connects to the receiver without an extension cable. The width of the current pulse is decreased on longer power cable because of $R_{P 2} .60 \mathrm{~m}$ and $100 \mathrm{~m}$ data indicate the error of pulse width increases on the longer cable (higher resistance).

This result follows proposed ECPLC communication model in Section III, because the pulse width decreasing is caused only by the resistance of the cable. i.e., Inductance and capacitance are negligible.

We inserted a variable resistance between the extension cable and changed $R_{P 2}$ intentionally so as to measure success rate versus resistance as shown at Fig. 7(b). There is no major difference between the two receivers. The result shows that communication success rate has rapidly decreased from around $1.1 \Omega$. This value corresponds to the cable length between $100 \mathrm{~m}$ to $200 \mathrm{~m}$.

\section{Communication with various plug-load devices}

Fig. 8 shows current waveforms from the ECPLC transmitter with four different plug-load devices which are $\mathrm{AC}$ motor, filament bulb, vacuum cleaner and AC/DC switching converter without the extension cable, $R_{P 2}$. Purple line shows the voltage on the AC power line and blue lines show current flows, $I_{C}$. The current pulses of ECPLC are modulated at around voltage zero crossing timing.

The four devices are chosen to investigate ECPLC characteristics with various plug-load devices power consumption pattern. In order to examine characteristics of low power factor device, a $70 \mathrm{~W}$ AC motor (Motor) is selected. The AC motor has mechanical impedance and the magnetic thing inside it

and the power factor is around $60 \%$ to $80 \%$. To evaluate with the nonlinear, conventional consumer electronics device, an $\mathrm{AC} / \mathrm{DC}$ switching converter (ACDC Con.) with a resistor is tested. Quite a few numbers of consumer electronics device require DC power and these devices use the converter. For evaluation with huge power consuming device, a filament bulb (Bulb) and a vacuum cleaner (Vacuum) are selected.

We evaluate success rates versus pulse width and height with the four plug-load devices and no device as shown as Fig. 9. The NL series means that there is no plug-load device, i.e. direct connection of the transmitter and the receivers. Both receivers get transmitted data correctly even the low power factor device or the AC/DC switching converter, with the default parameters, $T_{d}=250$ microseconds, $T_{w}=350 \mathrm{mi}-$ croseconds and $I_{h}=5.5 \mathrm{~A}$. Moreover, with lower pulse height around $2 \mathrm{~A}$, the normal receiver obtains data correctly. However the simple receiver does not collect enough data with the vacuum cleaner or with lower pulse height. The receiving process in the receiver is compromised because of vibrations of load current of the devices. For instance, the vibration of the vacuum cleaner is quite huge around $\pm 4 \mathrm{~A}$. The process tries to catch $4.62 \mathrm{~A}(70 \%$ of $5.5 \mathrm{~A})$ up/down edge of the pulse. The threshold value is nearly closed to the vibration, therefore the receiving process does fail. With lower pulse height, the process is also damaged because of the same reason. The normal receiver only works on zero crossing points and, thus, can avoid the vibrations.

\section{DISCUSSION AND FUTURE WORK}

Many-to-one connection is a future work of this research. In this paper, we tested the ECPLC system on the one-to-one connection between the transmitter and the receiver in order to evaluate characteristics. On the deployment of the power metering device, there are some granularity levels, which are smart power strips, smart outlets and smart meters. All plugload devices monitored by an individual smart power strip are the novel scenario and the scenario is just one-to-one connections. However, in the practical environment, smart power strips and smart outlets are extended by conventional power strips and some plug-load devices connect to one receiver and the connection must be many-to-one. ECPLC needs a method of collision management to treat the many-to-one connection.

Deep analysis and design of the detailed electric model of communication is surely important tasks. This paper introduces new communication mechanisms and provides some results based on the prototype to prove our concept. To discuss ECPLC mechanism more deeply, detailed electric model and new definitions of communication perspective are required. For example, to draw a graph of BER (Bit Error Rate) versus noise level requires a definition of noise of ECPLC.

ECPLC receiver may be implemented on conventional smart metering devices from the results of the experimentation because the pulse width is around 100 microseconds and the width is enough to measure by conventional ADC sampling rate. Considerations of developing the ECPLC communication system with the conventional deployed smart metering system is also a fancy future work. 


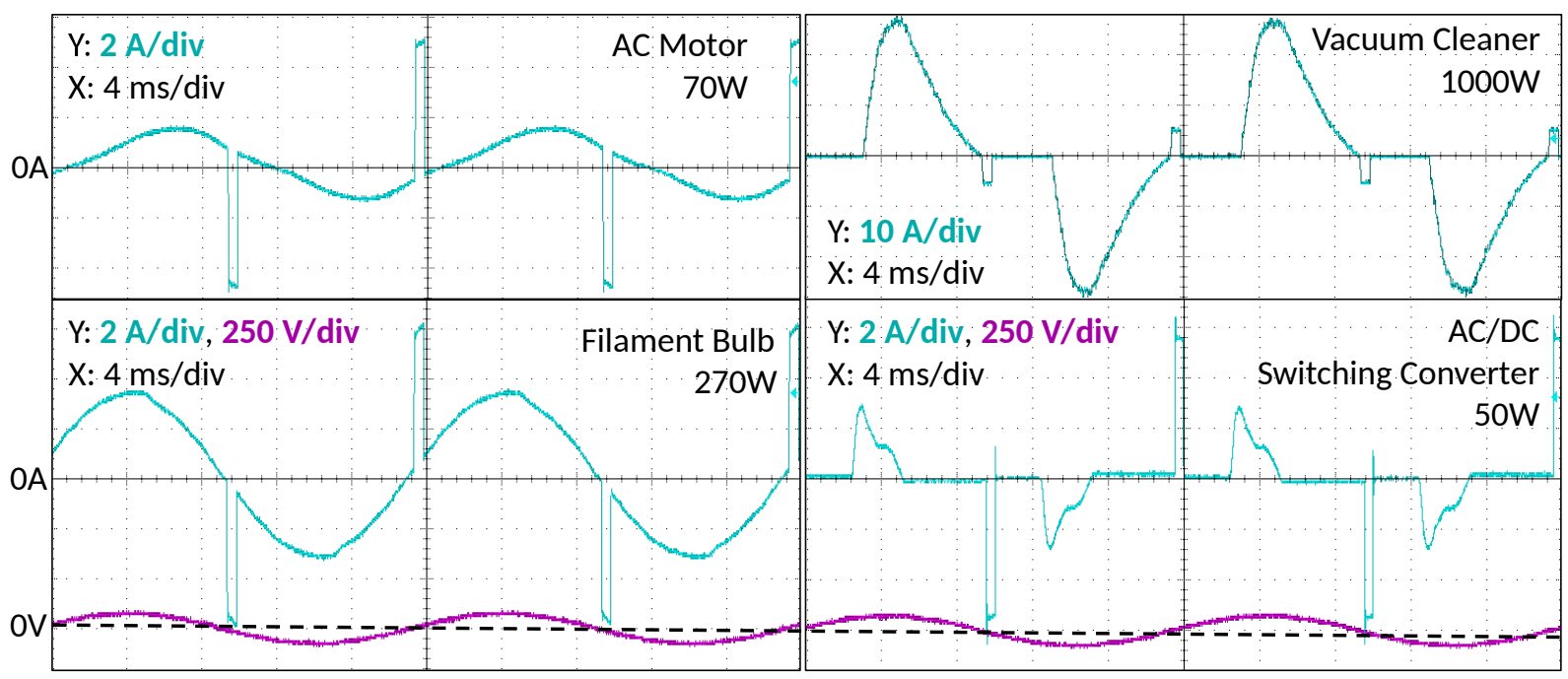

Fig. 8. ECPLC current pulse on plug-load devices current flows; Four images are screenshots from an oscilloscope (model: Tektronix MDO3014) and the series is centered by a downside edge of a pulse. The y-axis of the top-right one is different with others.

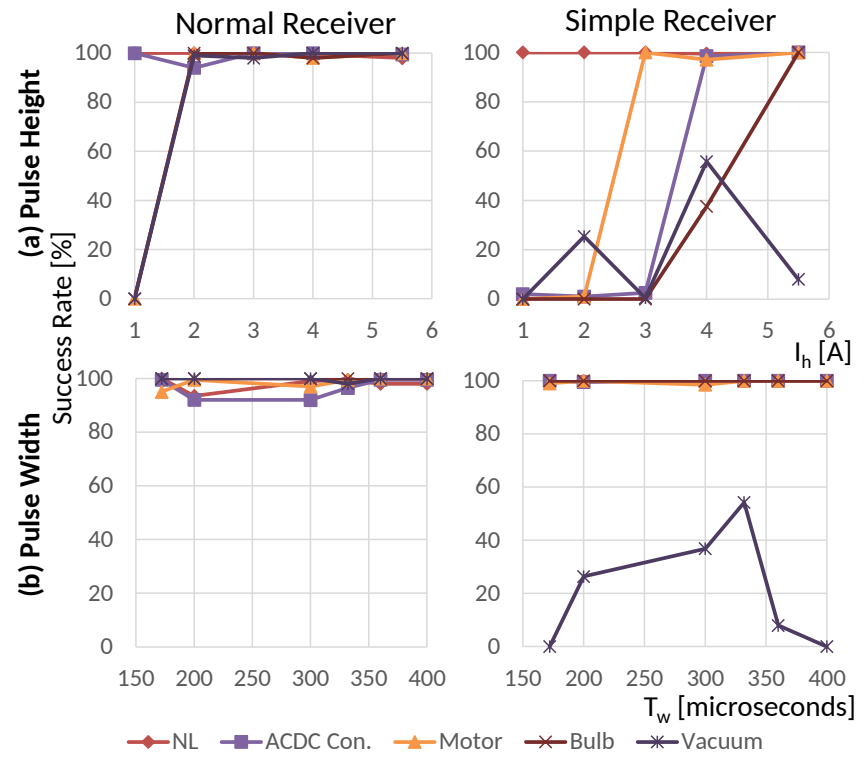

Fig. 9. Success rate versus pulse width and height: (a) pulse height, $I_{h}$, and $T_{w}$ is fixed to 350 microseconds; (b) pulse width, $T_{w}$, and $I_{h}=5.5 \mathrm{~A}$.

\section{CONCLUSION}

This paper presents electric current based power line communication (ECPLC). Feature of ECPLC is propagation range - transmitted signals limited on the connected smart power meters against conventional voltage based power line communication (EVPLC). The experimentation with working prototype shows ECPLC works well and characteristics are suitable for typical building environments. Throughput of ECPLC is achieved at $100 \mathrm{bps}$, which is enough for the target application, plug-load device auto identification system. The result of the experimentation shows that plug-load device auto identification system with ECPLC is feasible.

\section{REFERENCES}

[1] Ieee standard for broadband over power line networks: Medium access control and physical layer specifications. IEEE Std 1901-2010, pages 1-1586, Dec 2010.
[2] Sony develops " authentication outlet" where electricity use can be managed and consumed on a per-user and per-devicee basis. http:// www.sony.net/SonyInfo/News/Press/201202/12-023E/index.html, 2012. [Online; accessed 21-May-2015].

[3] S. Barker, M. Musthag, D. Irwin, and P. Shenoy. Non-intrusive load identification for smart outlets. In Smart Grid Communications (SmartGridComm), 2014 IEEE International Conference on, pages 548553, Nov 2014.

[4] Takekazu Kato, H. Cho, Dongwook Lee, T. Toyomura, and T. Yamazaki. Appliance recognition from electric current signals for informationenergy integrated network in home environments. Ambient Assistive Health and Wellness Management in the Heart of the City, pages 150 157, 2009.

[5] Sioe T. Mak and Thomas G. Moore. Twacs, a new viable twoway automatic communication system for distribution networks. part ii: Inbound communication. Power Engineering Review, IEEE, PER4(8):51-51, Aug 1984.

[6] Sioe T. Mak and Don L. Reed. Twacs, a new viable two-way automatic communication system for distribution networks. part i: Outbound communication. Power Apparatus and Systems, IEEE Transactions on, PAS-101(8):2941-2949, Aug 1982.

[7] N. Pavlidou, A.J.H. Vinck, J. Yazdani, and B. Honary. Power line communications: state of the art and future trends. Communications Magazine, IEEE, 41(4):34-40, April 2003.

[8] K. Razazian, M. Umari, A. Kamalizad, V. Loginov, and M. Navid. G3-plc specification for powerline communication: Overview, system simulation and field trial results. In Power Line Communications and Its Applications (ISPLC), 2010 IEEE International Symposium on, pages 313-318, March 2010

[9] Andreas Reinhardt, Paul Baumann, Daniel Burgstahler, Matthias Hollick, Hristo Chonov, Marc Werner, and Ralf Steinmetz. On the accuracy of appliance identification based on distributed load metering data. In Proceedings of the 2nd IFIP Conference on Sustainable Internet and ICT for Sustainability (SustainIT), pages 1-9, 2012.

[10] A. Ridi, C. Gisler, and J. Hennebert. A survey on intrusive load monitoring for appliance recognition. In Pattern Recognition (ICPR), 2014 22nd International Conference on, pages 3702-3707, Aug 2014.

[11] SAE. Sae j1772: Electric vehicle and plug in hybrid electric vehicle conductive charge coupler, January 2010.

[12] Ahmed Zoha, Alexander Gluhak, Muhammad Ali Imran, and Sutharshan Rajasegarar. Non-intrusive load monitoring approaches for disaggregated energy sensing: A survey. Sensors, 12(12):16838-16866, 2012 . 